\title{
Evaluation of neutrophil/lymphocyte ratio, platelet/lymphocyte ratio, mean platelet volume, and neutrophil/monocyte ratio in patients with benign breast lesions
}

\author{
Varman $\mathrm{A}^{1}$, Alkan $\mathrm{S}^{2}$ \\ Dr. Ali Kemal Belviranli Obstetrics and Pediatrics Hospital, General Surgery Department, \\ Fatih Mahallesi, Selcuklu, Konya, Turkey. alp.varman@gmail.com
}

\begin{abstract}
OBJECTIVES: In this study, we aimed to demonstrate the relationship between he neutrophil/lymphocyte ratio (NLR), platelet/lymphocyte ratio (PLR), neutrophil/monocyte rate (NMR) and mean platelet volume (MPV) in patients with BIRADS grades of benign breast lesions.

BACKGROUND: In many recent studies, NLR, PLR, NMR and MPV change significantly in connection with chronic inflammation and are suggested for use as prognostic markers in some diseases. However, the relationship between the cancer probability of benign breast diseases and mentioned hematological parameters has not been investigated in any previous study. We used the BIRADS classification to evaluate the malignancy probability of the cases.

METHODS: The hospital database of records of patients who were over 15 years of age and examined with bilateral ultrasonography between October 2019 and February 2020 were retrospectively scanned.

RESULTS: In total, 168 patients were included in the study. The average NLR and NMR values were higher in the BIRADS 2 group. There was no significant difference between the average PLR and MPV values of BIRADS groups.

CONCLUSIONS: Although some results seem to be statistically significant, there was no significant relationship between the hematological parameters we examined and the BIRADS grades of benign breast lesions (Tab. 2, Ref. 18). Text in PDF www.elis.sk

KEY WORDS: fibroadenoma, neutrophil lymphocyte ratio, breast ultrasonography.
\end{abstract}

\section{Introduction}

Benign breast lesions are very common today. Many women experience breast-related complaints that can occur at any time point of their lives and they come to hospital for medical checkup. The definition of benign breast lesions covers many various conditions, from a small disorder that may occur during the evolution of breast tissue to lesions that increase the risk of malignancy several times. For this reason, in 2009, ANDI classification was put forward for benign breast lesions. With this classification, the conditions are categorized relative to the stages of breast evolution as normal, disorder and disease. Lesions such as fibroadenoma, fibrocystic, or ductal ectasia, which fall into the class of disorders that do not have the potential of malignant transformation, while proliferative lesions containing atypia carry premalignant potential at varying rates (1). However, if the presence of benign

${ }^{1}$ Dr. Ali Kemal Belviranli Obstetrics and Pediatrics Hospital, General Surgery Department, Fatih Mahallesi, Secuklu, Konya, Turkey, and ${ }^{2}$ Bor Public Hospital, Chief Physician, General Surgery Department, Fatih Mahallesi, Secuklu, Konya, Turkey

Address for correspondence: A. Varman, MD, Dr. Ali Kemal Belviranli Obstetrics and Pediatrics Hospital, General Surgery Department, Fatih Mahallesi, Yeni Istanbul Cd. No: 30, 42285 Selcuklu, Konya, Turkey. Phone: +905548182334 breast lesion is detected upon imaging examinations for any reason, physical examination and imaging is recommended by calling the patient for periodic check-ups. The BIRADS (Breast Imaging Reporting and Data System) dictionary has been created for the purpose of using common language in the interpretation of imaging audit results (2). According to this classification, lesions are classified as follows:

Scores according to the BIRADS USG classification (3):

0 : Evaluation is inadequate, additional review is recommended.

1: Negative findings. The risk of malignancy is $0 \%$

2: Benign findings. $0 \%$ malignancy risk

3: Possibly benign. The risk of malignancy is about $0-2 \%$

4: Suspicious findings. A biopsy is recommended

4a: Low risk approx.: $2-10 \%$

4b: Moderate risk: $10-50 \%$

4c: High risk: $50-95 \%$

5: Very high risk: over $95 \%$

6: Proven malignancy

In many studies conducted in recent years, neutrophil/lymphocyte ratio (NLR), platelet/lymphocyte ratio (PLR), neutrophil/ monocyte ratio (NMR) and mean platelet volume (MPV) changed significantly relative to chronic inflammation and may be used as prognosis markers for some diseases. Owing to the fact that the results can be obtained by means of a simple and inexpensive 
blood, these parameters attract the attention of researchers. In the literature that we have researched, we found no study investigating the relationship between benign breast lesions and NLR, PLR, NMR and MPV. In this study, we aimed to reveal the relationship between benign breast lesions, BIRADS categories of these lesions and NLR, PLR, NMR and MPV. We anticipate that as the probability of malignancy increases along with the degrees of BIRADS, the examined hematological parameters, especially NLR, will be increasing as well.

\section{Methods}

We planned our study as a retrospective case-control study. The database of records of patients over 15 years of age and treated with bilateral breast ultrasonography (USG) between October 2019 and February 2020 at Dr. Ali Kemal Belviranli Obstetrics and Pediatric Hospital was retrospectively scanned. USG examinations of all patients were performed at the radiology department of our hospital; hemogram examinations were performed in our hospital's hematology laboratory. Age and gender, breast USG results, NLR, PLR, NMR and MPV values were recorded. Leukocytosis and malignancy were decided to be criteria for exclusion. Hematological data of cases with benign breast lesions (BIRADS 2, 3 and 4) and cases with no lesions (BIRADS 1) were compared. We expected the hematological parameters used as markers of chronic inflammation to be higher in cases with benign breast lesions.

\section{Statistical analysis}

SPSS package program version 20.0 (SPSS Inc, Chicago, IL, USA) was used for statistical analysis. When comparing the data across groups, the Kolmogorov-Smirnov test was used to check their suitability for normal distribution. When investigating the differences between the parametric data across the two groups, Student's t-test was used when the data were suitable for normal distribution, while Mann-Whitney U-test was employed when the data were not suitable for normal distribution. Pearson's correlation test was used when the data were suitable for normal distribution, and Spearman's correlation test was used when at least one data set was not suitable for normal distribution.

\section{Results}

A total of 168 patients who were requested for breast USG examination between October 2019 and February 2020 were in-

Tab. 1. Lesions and count.

\begin{tabular}{lccccc}
\hline & \multicolumn{5}{c}{ Count } \\
\cline { 2 - 6 } Type of Lesions & 1 & 2 & 3 & $\begin{array}{c}\text { More } \\
\text { than 3 }\end{array}$ & Total \\
\hline Fibrocyst & 13 & 8 & 5 & 31 & 57 \\
Fibroadenoma & 6 & 3 & 0 & 3 & 12 \\
Axillary lymphadenopathy & 3 & 2 & 0 & 0 & 5 \\
Fibrocyst and fibroadenoma & 0 & 1 & 1 & 11 & 13 \\
Ductal ectasia & 3 & 4 & 1 & 3 & 11 \\
Intramammary lymph node & 2 & 1 & 0 & 0 & 3 \\
Accessory breast & 0 & 2 & 0 & 0 & 2 \\
\hline
\end{tabular}

Tab. 2. Mean hematological parameter information and Kruskal-Wallis test results of BIRADS groups.

\begin{tabular}{lccccc}
\hline & BIRADS 1 & BIRADS 2 & BIRADS 3 & BIRADS 4A & $\mathrm{p}$ \\
\hline NLR & 1.86 & 2.20 & 1.85 & 1.61 & 0.011 \\
NMR & 9.50 & 10.63 & 9.98 & 7.67 & 0.043 \\
MPV & 9.44 & 9.35 & 9.31 & 8.80 & 0.59 \\
PLR & 12.73 & 13.31 & 11.93 & 13.83 & 0.098 \\
\hline
\end{tabular}

NLR - Neutrophil/lymphocyte ratio NMR: Neutrophil/monocyte ratio, MPV-Mean platelet volume PLR: Platelet/lymphocyte ratio

cluded in the study. All cases were female. Average age was 46.7 y (15-78). The Pearson correlation analysis revealed that the NLR value decreased along with the increase in age (correlation coefficient $=-0.228 ; \mathrm{p}=0.003$ )

Pathological USG results were found in 107 patients (BIRADS 2, 3 or 4A), while 61 patients had no pathological findings on ultrasonography (BIRADS 1). The mean age of those with pathological lesions on USG was $43.93 \mathrm{y}$, and the average age of those without pathological lesions was $49.82 \mathrm{y}$. The average age difference between these two groups was statistically significant (p: 0.003), while 50 cases were assessed as BIRADS 2, 54 as BIRADS 3 , and 3 as BIRADS 4A.

As a result of USG examinations, 57 patients had fibrocysts, 12 patients had fibroadenomas, 13 patients had a combination of fibrocysts and fibroadenomas. The detected lesions and the count of lesions are given in Table 1. There was no significant difference between the types of lesions and NLR, NMR, PLR, and MPV of the patients (p: 0.312, p: 0.068, p: 0.427, and p: 0.83, respectively).

In the study of the relationship between hematological parameters and degrees of BIRADS, there was a significant difference between the levels of NLR and NMR values and BIRADS degrees (Tab. 2). A post-hoc analysis showed that this difference for NLR was between the groups of BIRADS 2 and BIRADS 3 (p: 0.039). As for NMR, there was a difference between BIRADS 4A and other BIRADS groups (p: 0.05, p: 0.002, p: 0.015). In the USG study, there were no significant differences in hematological parameters (NLR, NMR, PLR, and MPV) between those with a lesion (BIRADS 2, 3 and 4A) and those without a lesion (BIRADS 1) (p: 0.21, p: 0.097, p: 0.92, and p: 0.374, respectively).

\section{Discussion}

Benign breast lesions are quite common among women. Among these lesions, fibroadenoma is the most common, while being present in $25 \%$ of asymptomatic women in the community (4). It is thought that approximately half of women over the age of 30 develop benign breast cysts, and only $20 \%$ of them are symptomatic (5). In a study conducted in China, it was shown that anxiety and depression symptoms were more common in women diagnosed with benign breast lesions which had a negative impact on their quality of life (6). Mastalgia has been associated with increased estrogen activity and presence of fibroadenoma (7). In addition, the patient, who notices a palpable mass in their breast, experiences anxiety at a level that affects their daily activities until the imaging tests are concluded and the physician is convinced that there is no cancer (6). 
NLR and PLR have been used as markers of chronic inflammation in many studies. After the 2001-study had introduced NLR as a cheap and simple inflammation parameter in critical patients, numerous studies paying attention to the relationship between NLR and chronic inflammation were published (8). A study involving 66 patients who were followed up for inflammatory bowel disease, showed that there was a strong relationship between high NLR and active disease (9). In another study, high NLR and PLR values were found to be associated with high mortality in acute ischemic stroke patients (10). Patients suffering from cardiovascular, cerebrovascular, and respiratory diseases, chronic renal failure, bowel diseases, rheumatologic diseases, diabetes and various cancers were observed to have an increase in MPV. It has been noted that MPV decreases along with exacerbation of ulcerative colitis, SLE and tuberculosis $(11,12)$. When we studied the literature, we found out that although there were many studies related to hemogram parameters and chronic inflammatory diseases, follow-up of malignant diseases, and their prognosis, there was no study investigating the relationship of hemogram parameters with benign breast lesions. We aimed at filling the latter gap in literature by publishing the results of a study on this subject.

In the study conducted by Cordiner et al. which included 707 early breast CA cases, it was found that NLR and PLR were not effective in predicting the prognosis (13). In a meta-analysis including 8,563 cases, it was shown that high NLR was associated with both low overall survival and low disease-free survival (14). In a study conducted on a Spanish population, higher NLR and PLR were found to be associated with worse overall and diseasefree survival (15). In another meta-analysis involving over 5,000 cases, high PLR was found to be associated with poor prognosis in patients with estrogen/progesterone receptor (ER/PR)-positive breast cancer. In the same study, no significant relationship was found between PLR and prognosis in patients with negative ER and PR (16). In our study, no significant relationship was found between the BIRADS categories of the cases and PLR.

A retrospective study of 150 breast cancer patients who had undergone neoadjuvant chemotherapy before surgery, found that poor prognosis was associated with high NLR. In the same study, it was stated that low NMR and better survival were found to be correlating, although it was not statistically significant (17). In our study, a significant relationship was found between NLR and NMR and BIRADS categories. Average NLR value was 1.95. While the highest mean NLR was 2.2, which was observed in BIRADS 2 cases, the lowest mean NLR was 1.61, which was seen in BIRADS 4A cases. The average NMR value of all our cases was 9.95. While the highest mean NMR value was 1.62, which was seen in BIRADS 2 cases, the lowest mean NMR was 7,67 and it was seen in BIRADS $4 \mathrm{~A}$ cases. Average NLR and NMR values of BIRADS categories are shown in Table 2. The post-hoc analysis revealed that the NLR values in BIRAD 2 group were significantly different from those in BIRADS 3 groups. Although the lowest value for both parameters was in the BIRADS 4A group, the lack of statistically significant difference in the NLR parameter as compared with the other groups can be explained by the very low number of cases ( 3 cases) in the BIRADS 4A group. It was not an expected result that NMR in the
BIRADS 4A group was significantly lower than in other groups, and NLR values were higher in BIRADS 2 cases than in BIRADS 3 cases. On the contrary, we expected the mean NLR and NMR values to increase along with the increase in the probability of malignancy and thus to reach their highest values in the BIRADS 4A group. In this respect, the conclusion of the study took us by surprise.

In our study, no significant relationship was found between the BIRADS categories of the cases and MPV.

In a previous study, it was found that NLR increases with age. In order to determine the reference values of NLR according to age groups, laboratory data of more than 70,000 patients were scanned and it was shown that NLR increased as the age progressed, however this trend was observed only in younger than middle-aged patients; at older age the values of NLR hit their plateau (18). These results are inconsistent with those of our study as we have found a negative correlation between the age of our patients and NLR. In the aforementioned study, it was stated that NLR kept increasing while the patients were still in their twenties, then struck a plateau that was observable in patients up to their sixties and finally, after the age of 60 , it continued to be increasing again. In our study, $95.8 \%$ $(161 / 168)$ of the cases were under the age of 65 years and $97.6 \%$ $(164 / 168)$ were over 20 years of age. The discrepancy in results could be probably caused by the fact that most of our cases were thus in the age category associated with the plateauing of NLR.

\section{Conclusion}

We examined some hematological data that provide useful information in terms of follow-up and prognosis in many cases of chronic inflammatory diseases, malignancies as well as cases with benign breast lesions that were followed up by breast USG. Contrary to our estimates, NMR value in BIRADS 4A was found to be significantly lower despite the fact that BIRADS 4A group has the highest probability of malignancy as compared to other groups. Again, contrary to our expectations, we found that NLR values in BIRADS 2 cases without malignancy were higher than those in BIRADS 3 cases with the malignancy probability of 2-10\%. We also found no significant relationship between MPV and PLR values and BIRADS categories. Similarly, we found no significant relationship between the types of lesions and investigated hematological parameters. In conclusion, there is no significant relationship between NLR, NMR, PLR and MPV values with the increase in BIRADS grades and malignancy risk in patients with the pre-diagnosis of benign breast lesions. Even though the results of our study were not significant in terms of follow-up and prognosis, we believe that we have contributed to the literature on a subject that has not been studied before.

\section{References}

1. Brunicardi FC. Schwartz Principles of Surgery, 9th edition. Meme. McGraw-Hill; 2010. 483-486

2. American College of Radiology. Breast imaging and Data System BI-RADS, 5th edition, D'Orsi CJ, Sickles EA, Mendelson EB, Morris EA Reston VA 2013. 
489-492

3. Venkataraman S, Slanetz PJ. BIRADS score Up to date February 2015; 8: 58.

4. Stachs A, Stubert J, Reimer T, Hartmann S. Benign Breast Disease in Women. Dtsch Arztebl Int 2019; 116 (33-34): 565-574. DOI: 10.3238/ arztebl.2019.0565. PMID: 31554551.

5. Stachs A, Stubert J, Reimer T, Hartmann S. Benign Breast Disease in Women. Dtsch Arztebl Int 2019; 116 (33-34): 565-574.

6. Lou Z, Li Y, Yang Y, Wang L, Yang J. Affects of Anxiety and Depression on Health-Related Quality of Life among Patients with Benign Breast Lumps Diagnosed via Ultrasonography in China. Int J Environ Res Publ Health 2015; 12 (9): 10587-601. DOI: 10.3390/ijerph120910587.

7. Tahir MT, Shamsudeen S. Mastalgia. 2020 Sep 19. In: StatPearls (Internet). Treasure Island (FL): StatPearls Publishing; 2020 Jan.

8. Zahorec R. Ratio of neutrophil to lymphocyte counts - rapid and simple parameter of systemic inflammation and stress in critically ill. Bratisl Med J 2001; 102 (1): 5-14.

9. Acarturk G, Acay A, Demir K, Ulu MS, Ahsen A, Yuksel S. Neutrophil-to-lymphocyte ratio in inflammatory bowel disease-as a new predictor of disease severity. Bratisl Med J 2015; 116 (4): 213-217.

10. Ozgen E, Guzel M, Akpinar CK, Yucel M, Demir MT, Baydin A. The relationship between neutrophil/lymphocyte, monocyte//lymphocyte, platelet/lymphocyte ratios and clinical outcomes after ninety days in patients who were diagnosed as having acute ischemic stroke in the emergency room and underwent a mechanical thro. Bratisl Med J 2020; 121 (9): 634-639.

11. Chu SG, Becker RC, Berger PB et al. Mean platelet volume as a predictor of cardiovascular risk: a systematic review and meta-analysis. J Thromb Haemostasis 2010; 8 (1): 148-156. DOI: 10.1111/j.15387836.2009.03584.x.
12. Korniluk A et al. Mean Platelet Volume (MPV): New Perspectives for an Old Marker in the Course and Prognosis of Inflammatory Conditions. Mediators Inflamm 2019; 9213074. DOI: 10.1155/2019/9213074.

13. Cordiner RL, Mansell J, Obondo C et al. Pre-operative haematological markers and prognosis in early breast cancer. Cancer Res 2014; 71 (Suppl 24): P2-12-30-P2-12-30. DOI: 10.1158/0008-5472.SABCS11-P2-12-30.

14. Ethier JL, Desautels D, Templeton A, Shah SP Amir E. Prognostic role of neutrophil-to-lymphocyte ratio in breast cancer: a systematic review and meta-analysis. Breast Cancer Res 2017; 19: 2. DOI: 10.1186/ s13058-016-0794-1.

15. Ramos-Esquivel A, Rodriguez-Porras L, Porras J. Neutrophil-lymphocyte ratio and platelet-lymphocyte ratio as prognostic factors in nonmetastatic breast cancer patients from a Hispanic population. Breast Dis 2017; 37: 1-6. DOI: 10.3233/BD-160251.

16. Zhang M, Huang XZ, Song YX, Gao P, Sun JX, Wang ZN. High platelet-to-lymphocyte ratio predicts poor prognosis and Clinicopathological characteristics in patients with breast Cancer: a meta-analysis. Biomed Res Int 2017; 2017: 9503025.

17. Marín Hernández C, Piñero Madrona A, Gil Vázquez PJ, Galindo Fernández PJ, Ruiz Merino G, Alonso Romero JL, Parrilla Paricio P. Usefulness of lymphocyte-to-monocyte, neutrophil-to-monocyte and neutrophil-to-lymphocyte ratios as prognostic markers in breast cancer patients treated with neoadjuvant chemotherapy. Clin Transl Oncol 2018; 20: 476-483. DOI: 10.1007/s12094-017-1732-0.

18. Aydin I et al. Farkli yaş gruplarinda nötrofil/lenfosit orani referans araliklari, Gülhane Askeri Tip Akademisi 2015. DOI: 10.5455/gulhane. 166398.

Received December 7, 2020. Accepted December 22, 2020. 